\title{
Multidetector computed tomographic characteristics of nonabsorbable polymer versus titanium ligature clips in a vascular model
}

Gary Foster, MD, ${ }^{\text {a,b }}$ Michael Plunkett, BS, ${ }^{\mathrm{a}}$ Aaron Wagner, BS, ${ }^{\mathrm{a}}$ Selwin Abraham, MD,,${ }^{\mathrm{a}}$ Duane Baldwin, MD, ${ }^{\mathrm{a}}$ Bryan Hill, BS, ${ }^{\mathrm{a}}$ and Hannah Kim, BS ${ }^{\mathrm{a}}$

Objective: Multidetector cardiac computed tomography is commonly performed to evaluate coronary bypass grafts, but titanium clips result in significant image artifact. Multidetector cardiac computed tomographic characteristics of newly developed nonabsorbable polymer clips are unknown. This study was undertaken to compare the image characteristics of polymer clips and titanium clips applied to a vascular model.

\begin{abstract}
Methods: A vascular model was created with two porcine internal thoracic arteries. Branches were ligated with 5 titanium clips on one vessel and 6 polymer clips on the other. Vessels were imaged under pressure with normal saline solution in a 16-detector computed tomographic scanner. Image intensity was quantified in absolute Hounsfield units for clips and adjacent lumen and then normalized to the average lumen intensity.
\end{abstract}

Results: No difference in absolute intensity was found between polymer clips and adjacent lumen (polymer clip $1021.2 \pm 19.0$ absolute Hounsfield units, adjacent lumen $1001.7 \pm 15.7$ absolute Hounsfield units, $P=.095$ ). A statistically significant difference was noted between titanium clips and adjacent lumen (titanium clips $3408.8 \pm$ 177.3 absolute Hounsfield units, adjacent lumen $1072.7 \pm 52.1$ absolute Hounsfield units, $P<.0001)$. A statistically significant difference was also noted between titanium and polymer clips $(P<.0001)$.

Conclusion: The use of polymer clips in coronary bypass grafts should result in significantly improved multidetector cardiac computed tomographic image quality.

Multidetector cardiac computed tomography (MDCT) is widely used as an alternative to invasive angiography to evaluate coronary bypass grafts. MDCT has several advantages relative to the current standard of invasive angiography, leading to its growing popularity with clinicians and patients. These advantages include short examination times, noninvasive acquisition of images, and high-resolution 3-dimensional images of both the vessel and lumen. ${ }^{1}$

Unfortunately, metal ligature clips used on coronary bypass grafts produce intense artifacts on computed tomography that are superimposed over the adjacent lumen, often making it impossible to analyze these regions of the vessel (Figure 1). ${ }^{2,3}$ Polymer clips have been used in a wide variety of operations and have been shown to have a reliability comparable to that of titanium clips. ${ }^{4}$ Currently, there is little information in the literature regarding nonabsorbable polymer clips and their effects on MDCT image quality. In this study, we compared the

From the Loma Linda University School of Medicine ${ }^{\mathrm{a}}$ and VA Loma Linda Health Care System, ${ }^{\text {b }}$ Loma Linda, Calif.

Received for publication Jan 4, 2008; revisions received April 3, 2008; accepted for publication May 13, 2008.

Address for reprints: Gary Foster, MD, Cardiology Section, VA Loma Linda Healthcare System (111C), 11201 Benton St, Loma Linda, CA 92357 (E-mail: Gary.

Foster2@va.gov).

J Thorac Cardiovasc Surg 2008; 136:1324-7

$0022-5223 / \$ 34.00$

Copyright (c) 2008 by The American Association for Thoracic Surgery doi:10.1016/j.jtcvs.2008.05.033
MDCT characteristics of titanium clips and polymer clips in a vascular model.

\section{MATERIALS AND METHODS}

An in vitro porcine vessel model was created. Two internal thoracic arteries (ITAs) were harvested from pig carcasses obtained from an animal research facility. The pigs had been killed as part of an institutional review board-approved protocol not related to this experiment. The ITAs were stored in refrigerated, isotonic saline solution until use. The side branches were ligated with ligature clips according to the diagram in Figure 2. We applied 5 titanium clips (Ethicon Ligaclip Extra LT100, $3 \mathrm{~mm} /$ small; Ethicon Endo-Surgery, Inc, Cincinnati, Ohio) onto side branches along the length of the first artery (Figure 3,B) and 6 polymer clips (Weck Hem-o-lok $3 \mathrm{~mm}$; Weck Closure Systems, Triangle Park, NC) onto the side branches along the length of the second artery (Figure 3, A). Both types of clips have been approved by the Food and Drug Administration and are available for routine use during surgery. Subsequently, an end of each artery was clamped, and the other end was attached to an intravenous fluid bag of normal saline solution that was pressurized enough to maintain a turgid system. The vessels were imaged with a Toshiba Aquilion 16 computed tomographic scanner (Toshiba Corporation Medical Systems Company, Tokyo, Japan) according to the following parameters: detector collimation $16 \times 0.5 \mathrm{~mm}$, tube rotation time $400 \mathrm{~ms}$, tube voltage $120 \mathrm{kV}$, tube current $200 \mathrm{~mA}$, pitch $4.30 \mathrm{~mm} /$ rotation, and $250 \mathrm{mAs}$.

\section{Data Collection and Analysis}

All image analyses were made with a Vital Images Vitrea 2 workstation (Vital Images, Inc, Minnetonka, Minn). Image intensity on MDCT was quantified in Hounsfield units (HU). ${ }^{5}$ The HU value was determined for each clip, its adjacent lumen, and the normal lumen, as shown in Figure 2. The image intensities for the lumen directly above and below a titanium clip and a polymer clip were determined and identified as 


\section{Abbreviations and Acronyms}

$\mathrm{HU}=$ Hounsfield unit

MDCT $=$ multidetector cardiac computed tomography the normal lumen, with which the clips and adjacent lumen could be compared for normalization. Ten separate HU measurements were determined for each location to determine average $\mathrm{HU}$ values for normal lumen, titanium clip, titanium clip-adjacent lumen, polymer clip, and polymer clipadjacent lumen.

By convention $1000 \mathrm{HU}$ were added to the averaged values for normal lumen, titanium clip, titanium clip-adjacent lumen, polymer clip, and polymer clip-adjacent lumen to ensure that all measurements were being compared on an absolute scale. An absolute scale was used for purposes of calculating percentages. To display image intensity on a histogram (Figure 4), we normalized titanium clip, titanium clip-adjacent lumen, polymer clip, and polymer clip-adjacent lumen values in relation to the normal lumen value. We determined normalized intensities for titanium clip, titanium clip-adjacent lumen, polymer clip, and polymer clip-adjacent lumen according to the following equation: Normalized intensity $=[($ Mean absolute intensity $)-($ Mean absolute normal lumen $)] /$ Mean absolute normal lumen. The normal lumen intensity for the titanium clip vessel was used to determine normalized values for all titanium clip measurements. Likewise, the normal lumen intensity for the polymer clip vessel was used to determine normalized values for all polymer clip measurements. A 2-tailed $t$ test was used to determine the statistical significance between groups.

\section{RESULTS}

MDCT scans of titanium and polymer clips applied to a vascular model are shown in Figure 5. The titanium clips show a considerable amount of artifact, manifested as a bloom extending into the adjacent lumen and conspicuous bright spots on the ITA. The polymer clips contributed no visually discernable artifact on the ITA and appear as faint spots adjacent to the arterial lumen.

Table 1 shows the absolute HU and normalized intensities of normal lumen for polymer clip, polymer clip, polymer

\section{1 - Clip \\ 2 - Adjacent Lumen 3 - Normal Lumen}

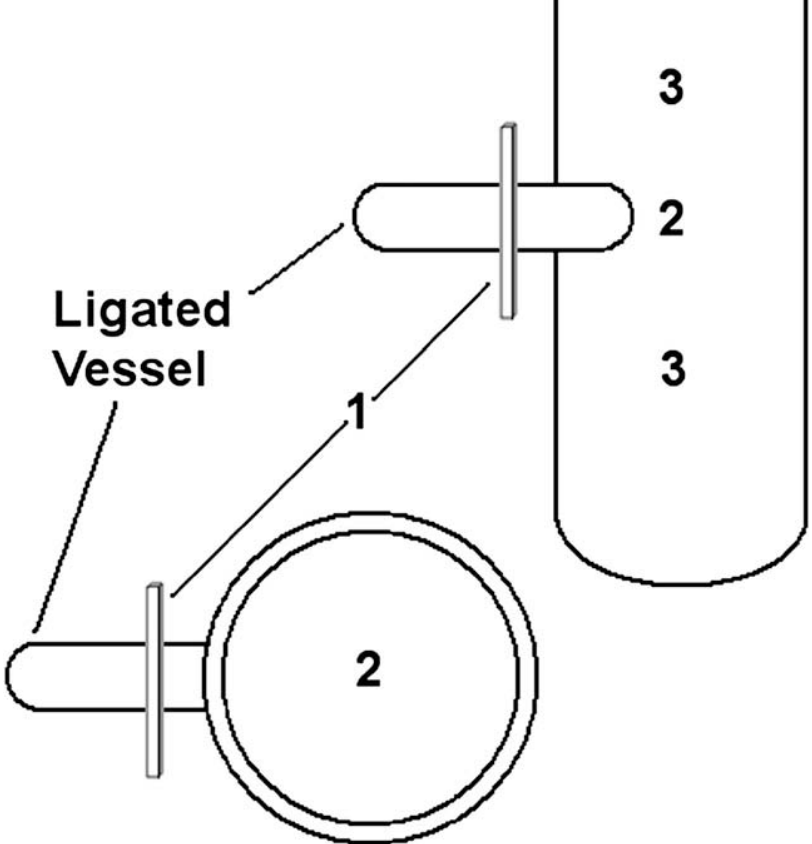

FIGURE 2. Diagram of porcine internal thoracic artery model. Branches were ligated with either titanium or polymer clips. Measurements were taken at clip (1), adjacent lumen (2), and above and below clip (3).

clip-adjacent lumen, normal lumen for titanium clip, titanium clip, and titanium clip-adjacent lumen. No statistically significant difference in absolute intensity was found between polymer clip and polymer clip-adjacent lumen (polymer clip $1021.2 \pm 19.0 \mathrm{HU}$, polymer clip-adjacent lumen $1001.7 \pm 15.7 \mathrm{HU}, P=.095)$. A statistically significant difference was noted between titanium clip and titanium
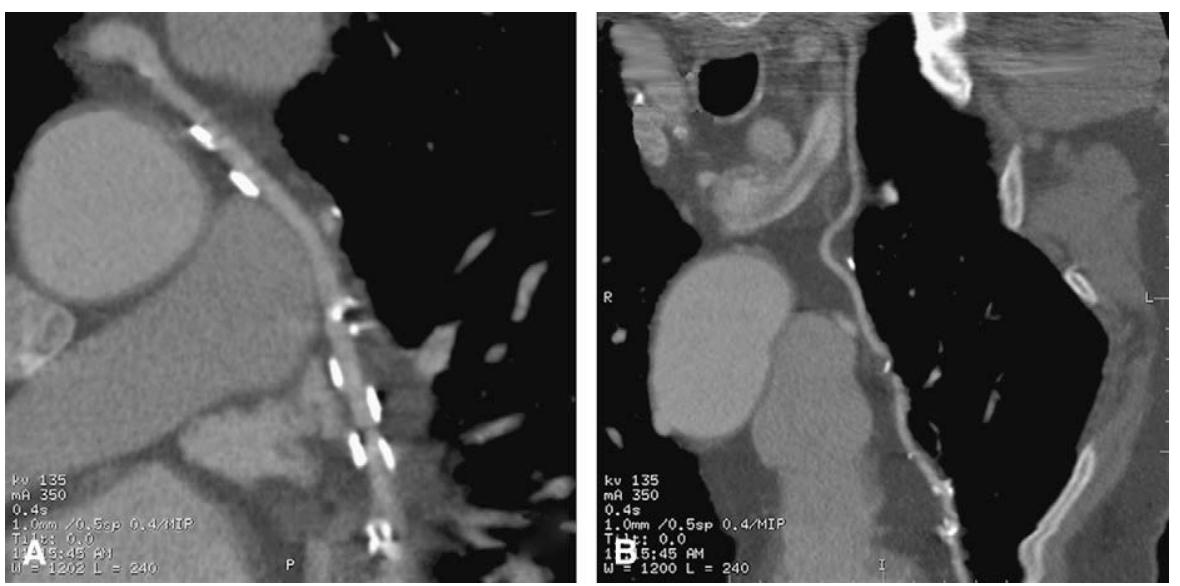

FIGURE 1. Clinical multidetector cardiac computed tomographic images of saphenous vein (A) and left internal thoracic artery (B) grafts in which titanium clips were used. 


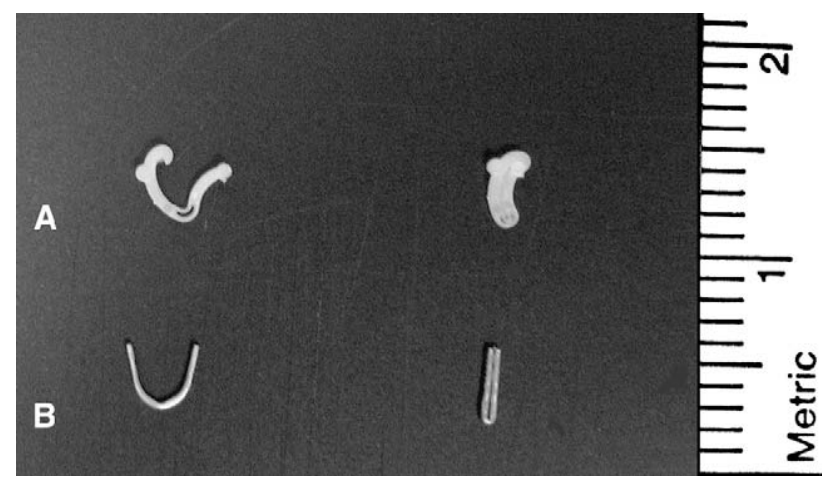

FIGURE 3. Polymer (A) and titanium (B) clips.

clip-adjacent lumen intensities (titanium clip $3408.8 \pm$ 177.3 HU, titanium clip-adjacent lumen $1072.7 \pm 52.1$ HU, $P<.0001)$. A statistically significant difference was also noted between the titanium clip and polymer clip intensities (titanium clip $3408.8 \pm 177.3 \mathrm{HU}$, polymer clip $1021.2 \pm 19.0 \mathrm{HU}, P<.0001)$. Whereas no significant difference was found between polymer clip-adjacent lumen and titanium clip-adjacent lumen absolute intensities (polymer clip-adjacent lumen $1001.7 \pm 15.7 \mathrm{HU}$, titanium clipadjacent lumen $1072.7 \pm 52.1 \mathrm{HU}, P=.068$ ), a difference was found between the normalized intensities, indicating that titanium clip-adjacent lumen was brighter than polymer

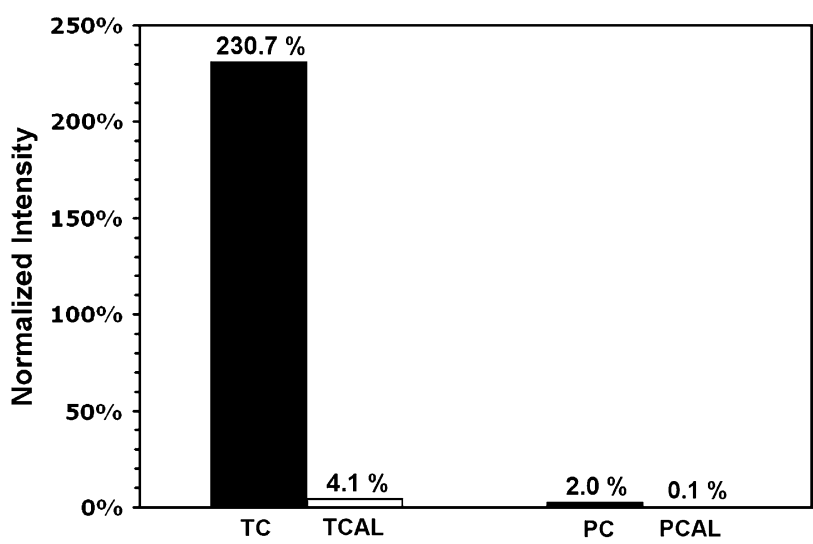

FIGURE 4. Normalized comparisons among image intensities of titanium clip, titanium clip-adjacent lumen, polymer clip, and polymer clip-adjacent lumen. $T C$, Titanium clip; $T C A L$, titanium clip-adjacent lumen; $P C$, polymer clip; $P C A L$, polymer clip-adjacent lumen.

clip-adjacent lumen when considering percentage increase of intensity relative to the normal lumen (titanium clip-adjacent lumen $4.1 \%$, polymer clip-adjacent lumen $0.1 \%$ ).

\section{DISCUSSION}

There are many concerns for the surgeon when selecting a technique to harvest the ITA, including speed, safety,
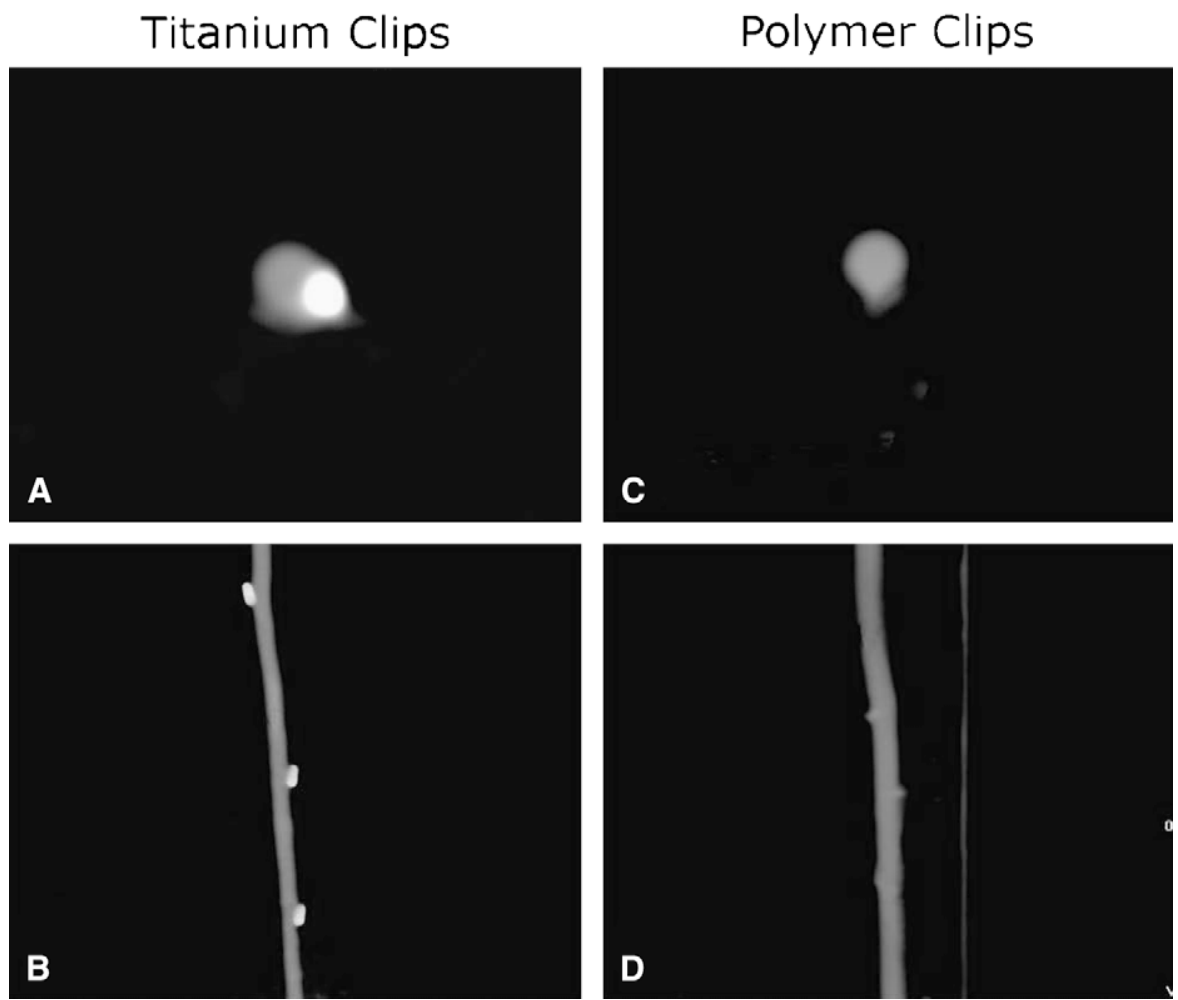

FIGURE 5. Multidetector cardiac computed tomography of porcine internal thoracic artery model showing cross-sectional (A) and longitudinal (B) views with titanium clips and cross-sectional (C) and longitudinal (D) views with polymer clips. 
TABLE 1. Relationship between intensity in absolute Hounsfield units and normalized intensity

\begin{tabular}{lccc}
\hline $\begin{array}{c}\text { Location on } \\
\text { internal } \\
\text { thoracic artery }\end{array}$ & No. & $\begin{array}{c}\text { Absolute Hounsfield } \\
\text { units (mean } \pm \text { SD) }\end{array}$ & $\begin{array}{c}\text { Normalized } \\
\text { intensity }(\%)\end{array}$ \\
\hline NL PC & 2 & $1000.9 \pm 3.4$ & $0.0 \%$ \\
PC & 6 & $1021.2 \pm 19.0 *$ & $2.0 \%$ \\
PC AL & 5 & $1001.7 \pm 15.7 \dagger$ & $0.1 \%$ \\
NL TC & 2 & $1030.8 \pm 7.5$ & $0.0 \%$ \\
TC & 5 & $3408.8 \pm 177.3 \ddagger$ & $230.7 \%$ \\
TC AL & 4 & $1072.7 \pm 52.1$ & $4.1 \%$ \\
\hline
\end{tabular}

$N L$, Normal lumen; $P C$, polymer clip; $A L$, adjacent lumen; $T C$, titanium clip. $* P<.001$ for polymer clip versus titanium clip. $\dagger$ Not statistically different for polymer clip versus adjacent lumen or titanium clip versus adjacent lumen. $\underset{\ddagger}{\ddagger} P<.001$ for titanium clip versus adjacent lumen.

security, and cost. This study was designed to evaluate another consideration, the long-term effect on the ability to image the grafted vessels.

MDCT is a valuable tool in assessing the patency of coronary grafts, but interpretation is routinely complicated by artifacts from ligature clips ${ }^{1,6}$ (Figures 1 and 5). Our results demonstrate that polymer clips do not produce clip-induced artifacts. In contrast, titanium clips produced intense artifacts that dramatically degrade image quality.

Jellison and colleagues ${ }^{4}$ determined that polymer clips are statistically more secure than titanium clips when applied to equine renal arteries. Polymer clips have a locking mechanism, making dislodgment of a properly applied clip unlikely. Although titanium clips have proved invaluable in the reliable ligature of vessels, multiple clinical cases of titanium clip dislodgment have been reported. ${ }^{4,7-12}$

One possible limitation to our study is the use of a static vascular model. Patient movement is thought to enhance the artifacts induced by surgical clips. Our study demonstrates the artifacts caused by the clip material alone. The clip-induced artifact is a multifaceted phenomenon that is also influenced to variable degrees by patient movement, respiration, intestinal peristalsis, and pulsatile vascular motion. ${ }^{3}$ Additionally, our vascular model was surrounded by air, unlike a living model surrounded by tissues of varying densities. Bone, blood, and respiratory tissue have unique image characteristics that themselves may introduce artifact and reduce image quality. Comparison of MDCT images of a patient with an ITA graft clipped with titanium clips, however, shows significant similarity to our static vascular model (Figures 1 and 5).
In our study, titanium clips produced much more artifact than polymer clips, both subjectively and quantitatively. MDCT has made it possible to determine the patency of vascular grafts by evaluating the image intensity of a lesion relative to the vessel lumen. In cases where artifacts prevent proper imaging of the vessel lumen, invasive angiography may be the only option for determining coronary graft patency.

\section{CONCLUSION}

Polymer clips do not cause significant image artifacts on MDCT in a vessel model. Use of polymer clips during coronary artery bypass grafting would facilitate postoperative assessment of vascular grafts with MDCT, lower the risk of misinterpretation, and allow many patients to avoid unnecessary invasive angiographic procedures.

We are grateful to Guilda Sarraf, MD, and Ted Millian, CRT, for facilitating the development of the vascular model and acquiring MDCT images, and to Floyd Petersen for assisting in statistical analysis.

\section{References}

1. Chartrand-Lefebvre C, Cadrin-Chênevert A, Bordeleau E, Ugolini P, Ouellet R, Sablayrolles JL, et al. Coronary computed tomography angiography: overview of technical aspects, current concepts, and perspectives. Can Assoc Radiol J. 2007;58:92-108.

2. Marks WM, Callen PW. Computed tomography in the evaluation of patients with surgical clips. Surg Gynecol Obstet. 1980;151:557-9.

3. Glover GH, Pelc NJ. An algorithm for the reduction of metal clip artifacts in CT reconstructions. Med Phys. 1981;8:799-807.

4. Jellison FC, Baldwin D, Berger KA, Maynes LJ, Desai PJ. Comparison of nonabsorbable polymer ligating and standard titanium clips with and without a vascular cuff. J Endourol. 2005;19:889-93.

5. Hounsfield GN. Nobel award address. Computed medical imaging. Med Phys 1980;7:283-90.

6. Gross SC, Kowalski JB, Lee SH, Terry B, Honickman SJ. Surgical ligation clip artifacts on CT scans. Radiology. 1985;156:831-2.

7. Nelson MT, Nakashima M, Mulvhill SJ. How secure are laparoscopically placed clips? An in vitro and in vivo study. Arch Surg. 1992;127: 718-20.

8. Riskin DJ, Schwaitzberg SD. A comparison of holding strength of various surgical clips. Surg Endosc. 2003; 17:654-6.

9. Baek M, Chun H, Oh SJ, Kim HH. Open conversion from laparoscopic nephrectomy: slippage of surgical clips ligating the renal artery affected by atherosclerosis. J Urol. 2004;171:333-4.

10. Maartense S, Heintjes RJ, Idu M, Bemelman FJ, Bemelman WA. Renal artery clip dislodgement during hand-assisted laparoscopic living donor nephrectomy. Surg Endosc. 2003; 17:1851

11. Martay K, Dembo G, Vater Y, Charpentier K, Levy A, Bakthavatsalam R. Unexpected surgical difficulties leading to hemorrhage and gas embolus during laparoscopic donor nephrectomy: a case report. Can J Anaesth. 2003; 50:891-4.

12. Hsu TH, Su LM, Ratner LE, Kavoussi LR. Renovascular complications of laparoscopic donor nephrectomy. Urology. 2002;60:811-5. 